\title{
The Galactic thick and thin disks: differences in evolution
}

\author{
T. V. Nykytyuk ${ }^{1}$ and T. V. Mishenina ${ }^{2}$ \\ 1 Main Astronomical Observatory, Ak. Zabolotnoho St. 27, Kyiv 03680, Ukraine \\ e-mail: nikita@mao.kiev.ua \\ 2 Astronomical Observatory Odessa National University, Odessa, Ukraine
}

Received 21 March 2005 / Accepted 13 March 2006

\begin{abstract}
Context. Recent observations demonstrate that the thin and thick disks of the Galaxy have different chemical abundance trends and evolution timescales.

Aims. The relative abundances of $\alpha$-elements in the thick Galactic disk are increased relative to the thin disk. Our goal is to investigate the cause of such differences in thick and thin disk abundances.

Methods. We investigate the chemical evolution of the Galactic disk in the framework of the open two-zone model with gas inflow. Results. The Galactic abundance trends for $\alpha$-elements $(\mathrm{Mg}, \mathrm{Si}, \mathrm{O})$ and $\mathrm{Fe}$ are predicted for the thin and thick Galactic disks.

Conclusions. The star formation histories of the thin and thick disks must have been different and the gas infall must have been more intense during the thick disk evolution that the thin disk evolution.
\end{abstract}

Key words. Galaxy: evolution - Galaxy: abundances - Galaxy: disk

\section{Introduction}

Recent studies of the kinematics and ages of disk stars of our Galaxy have revealed the presence of two distinct populations in the Galactic disk, called the thick and the thin disk populations. Gilmore \& Raid (1983) have shown that the stellar density distribution towards the Galactic South Pole requires the presence of at least two kinematically distinct disk structures. For $z<1 \mathrm{kpc}$ their data are fitted by a single exponent and by a second exponent in the range $1-5 \mathrm{kpc}$ above Galactic plane. The authors considered this as evidence of the presence of an old thin disk and a thick disk. The thick disk stars have a scale height from 760 to 1450 pc (Gilmore \& Raid 1983; Robin et al. 1996; Reyle \& Robin 2001; Chen 1997); the scale height of the thin disk is 100 to 340 pc (Gilmore \& Raid 1983; Chen 1997). The velocity dispersion $\left(\sigma_{u}, \sigma_{v}, \sigma_{w}\right)$ of the thick and thin disk stars is also different; it is $(63 \pm 6,39 \pm 4,39 \pm 4) \mathrm{km} \mathrm{s}^{-1}$ and $(39 \pm 2$, $20 \pm 2,20 \pm 1$ ) correspondingly (Soubiran et al. 2003).

Jimenez et al. (1998) used the Hipparcos color-magnitude diagram of field stars and derived a minimum age for the Galactic disk of about 8 Gyr. Fuhrmann (1998) found that the thin disk stars in his sample are younger than 8-9 Gyr while the thick disk star ages exceed 10 Gyr. Bernkopf et al. (2001) suggested that the maximum age for the thin disk stars is near 9 Gyr whereas the thick disk stars have ages from 12 to 14 Gyr. Bensby et al. (2003) found that the thick disk stars are older on average than the thin disk stars, 11.2 \pm 4.3 Gyr and $4.9 \pm 2.8$ Gyr. In Feltzing et al. (2003) they derived slightly higher average ages $-12.1 \pm$ $3.8 \mathrm{Gyr}$ and $6.1 \pm 2.0 \mathrm{Gyr}$ for thick and thin disks correspondingly. Thus, the thin and thick disks were formed at different epochs.

The chemical characteristics of these two disk subsystems of the Galaxy are also noticeably different. A kinematic study of the local $\mathrm{G}$ dwarf metallicity distribution of our Galaxy provides evidence that the abundance distribution below $[\mathrm{Fe} / \mathrm{H}] \sim-0.4$ contains two overlapping distributions, the thin and the thick disks (Wyse \& Gilmore 1995). The mean metallicity of the thick disk population, $\langle[\mathrm{Fe} / \mathrm{H}]\rangle$, covers the range between -0.5 and -0.7 dex (Soubiran et al. 2003; Wyse \& Gilmore 1995; Robin et al. 1996) while the mean value of the thin disk star metallicity is -0.17 to -0.25 dex (Soubiran et al. 2003; Wyse \& Gilmore 1995).

The thick and thin disk abundance trends partly overlap in the range $-0.8<[\mathrm{Fe} / \mathrm{H}]<-0.4$ but they are different in $[\alpha / \mathrm{Fe}]$. Gratton et al. (1996) pointed out that changes in $[\mathrm{Fe} / \mathrm{O}]$ values of the disk stars can be explained by a systematic difference in the chemical composition of the thin and thick disk stars. Later Gratton et al. (2000) studied this interpretation in detail; as their $[\mathrm{Fe} / \mathrm{O}]$ vs. $[\mathrm{O} / \mathrm{H}]$ diagram shows, there are two groups of disk stars with $[\mathrm{O} / \mathrm{H}]>-0.5-$ the thin disk stars with $[\mathrm{Fe} / \mathrm{O}]>-0.25$ and the thick disk stars with $[\mathrm{Fe} / \mathrm{O}]<-0.25$.

The thick disk has a higher $[\mathrm{O} / \mathrm{Fe}]$ ratio than the thin disk at sub-solar metallicities. The thick disk also shows signatures of chemical enrichment by type Ia supernovae (Bensby et al. 2004). Fuhrmann (1998) have demonstrated clear disk separation in $[\mathrm{Mg} / \mathrm{Fe}]$ in the range $-0.6<[\mathrm{Fe} / \mathrm{H}]<-0.3$ in their $[\mathrm{Mg} / \mathrm{Fe}]$ vs. $[\mathrm{Fe} / \mathrm{H}]$ diagram - the stars with thick disk kinematics have $[\mathrm{Mg} / \mathrm{Fe}] \simeq+0.4$ while the thin disk stars have $[\mathrm{Mg} / \mathrm{Fe}]$ value decreasing from +0.2 to 0.0 dex. Bensby et al. (2003) have analyzed the spectra of $F$ and $G$ disk dwarfs with metallicities located in the range $-0.8<[\mathrm{Fe} / \mathrm{H}]<+0.4$ and have found that the thin and thick disk abundance trends are clearly separated at $[\mathrm{Fe} / \mathrm{H}]<0$. Bensby et al. (2005) confirms their previous results - there are distinct and separate abundance trends between the thin and thick disks. The thick disk stars are more enhanced in their $\alpha$-element abundances than the thin disk at a given $[\mathrm{Fe} / \mathrm{H}]$ below solar metallicities. 
On the other hand, Chan et al. (2000) have studied the chemical composition of $90 \mathrm{~F}$ and $\mathrm{G}$ disk dwarfs and did not find a clear separation of $[\alpha / \mathrm{Fe}]$ vs. $[\mathrm{Fe} / \mathrm{H}]$ into thin and thick disks. As shown by Prochaska et al. (2000), this situation appears because the old high - metallicity thick disk stars with $T_{\text {eff }}<5700 \mathrm{~K}$ were not been included in the sample of dwarfs investigated by Chan et al. (2000).

Recent investigations showed that abundance trends for $\mathrm{Mn}$ and $\mathrm{Eu}$ are also different for these two disk components. In particular, a detailed study of the Mn abundance trend of the disk and metal-rich halo stars was carried out by Nissen et al. (2000). Prochaska \& McWilliam (2000) have revisited these data (with the new hyperfine structure of the $\mathrm{MnI}$ line) and have shown that thin disk stars with $-0.8<[\mathrm{Fe} / \mathrm{H}]<-0.2$ have $[\mathrm{Mn} / \mathrm{H}] \simeq$ -0.1 while thick disk stars $[\mathrm{Fe} / \mathrm{H}]<-0.6$ have $[\mathrm{Mn} / \mathrm{Fe}] \simeq-0.3$. Mashonkina \& Gehren $(2000,2001)$ report that a step-like change exists in $[\mathrm{Eu} / \mathrm{Ba}]$ and $[\mathrm{Ba} / \mathrm{Fe}]$ ratios at the the transition of the thick to thin disk. They also found that europium is overabundant relative to barium and iron in the halo and thick disk stars. Nearly solar $[\mathrm{Eu} / \mathrm{Fe}],[\mathrm{Ba} / \mathrm{Fe}]$ and $[\mathrm{Eu} / \mathrm{Ba}]$ ratios are found for thin disk stars.

In this paper, we consider the thick and thin disks as two separate components of the Galactic disk which have been formed at different epochs and have different evolutionary timescales.

Matteucci \& Greggio (1986) suggested that the evolution of the abundance of oxygen vs. iron can be explained by an origin of iron and oxygen from different SN types. Such an interpretation is widely accepted since provides an explanation for the observed overabundances of $\alpha$-elements in the halo. Iron is predominantly synthesized by type Ia SN (intermediate mass stars in binary systems), while $\alpha$ elements (in our case $\mathrm{O}, \mathrm{Mg}, \mathrm{Si}$ ) are synthesized by massive stars. Thus, abundance ratios depend on the stellar nucleosynthesis, stellar lifetimes and initial mass function. But the variation of abundance ratios as a function of metallicity or time depends on the star formation history as well. This allows us to use the variations of $[\alpha / \mathrm{Fe}]$ as an indicator of the star formation history (Matteucci 1992). Since the $[\alpha / \mathrm{Fe}] \mathrm{ra}-$ tios for the thick and thin disk stars are distinct, we make the assumption that star formation histories of the two disk components must be different. The aim of this paper is to find such parameters for the star formation history which allow us to reproduce the observed $\alpha$-element abundances in the thin and thick disks of the Galaxy at metallicities below the solar value.

This paper is organized as follows. In Sect. 2 we describe the model and its main components for the chemical evolution of the Galactic thin and thick disks. In Sect. 3 we show and discuss the model predictions for the abundances of elements considered $(\mathrm{Mg}, \mathrm{Si}, \mathrm{Fe})$ for the thick and thin Galactic disks. In Sect. 4 we summarize the main conclusions.

\section{The model}

Our model for the chemical evolution of the Galaxy is described in detail by Pilyugin \& Edmunds (1996) and Pilyugin (1993). We construct an open two-zone chemical evolution model of the Galactic disk in order to investigate the distinction in chemical characteristics of the Galactic disk subsystems. The Galactic disk is divided into two zones - the thin and thick disks; the mass of gas, heavy elements, stars and stellar remnants are computed as a function of time for each component. The gas infall from the extrahalo takes place during their evolution.

In our model the continuous star formation process in the galactic disk is considered as a sequence of bursts with a generation of stars formed during each burst ${ }^{1}$. Each stellar generation contributes to the chemical enrichment of the interstellar medium; the next stellar generation is formed from the galactic gas enriched by heavy elements ejected from the previous stellar generation. We set an interval between bursts of $50 \mathrm{Myr}$ as such a value is most suitable for a Galactic disk model. The number of bursts depends on the interval between bursts and the duration of disk evolution. Thus, during galactic evolution the heavy element fraction and number of stars increases while the galactic gas mass decreases. We have used use the Eqs. (3)-(5) from Pilyugin (1993) for a description of the temporal variations of the mass of gas, stars and heavy elements during galactic evolution. It is assumed that the evolution time of the disk of our Galaxy is 13 Gyr (Cowan et al. 1991) and the burst interval has been set to 50 Gyr (Pilyugin \& Edmunds 1996).

The star formation rate $\psi(t)$ in thick and thin disks is described in the following way (Pilyugin \& Edmunds 1996):

$\psi(t) \sim\left\{\begin{aligned} t \cdot \mathrm{e}^{-t / T_{\text {top }}}, & t \leq T_{\text {top }} \\ \mathrm{e}^{-t / T_{\text {sfr }}}, & t \geq T_{\text {top }}\end{aligned}\right.$

where $T_{\text {top }}$ and $T_{\text {sfr }}$ are free parameters of the star formation rate.

The star formation history is described by a set of star formation bursts whose amplitudes are derived as

$M_{\mathrm{b}_{i}}=\int_{t_{\mathrm{b}_{i-1}}}^{t_{\mathrm{b}_{i}}} \psi(t) \mathrm{d} t$,

where $t_{b_{i}}$ and $t_{b_{i-1}}$ are the times of beginning of $j-$ and $j-1$ star formation bursts. The interval between consistent star formation bursts is $50 \mathrm{Myr}$.

We also assume that infall of intergalactic gas takes place on the disk during the galaxy's lifetime. According to Pilyugin \& Edmunds (1996), the infall rate the is described by the function

$A(t)=a_{0} \mathrm{e}^{-t / T_{\text {inf }}}$

where $T_{\text {inf }}$ and $a_{0}$ are free model parameters. The infalling gas has the primordial chemical composition since, according to Tosi (1988), the accretion of a gas with metallicity up to 0.1 solar abundance gives the same result as accretion of a gas with primordial chemical composition.

The star formation histories of the disk components were chosen so that the thick disk stars were formed 10-13 Gyr ago while the majority of the thin disk stars would have ages less than 10 Gyr.

\subsection{Nucleosynthesis}

The abundance of the elements synthesized by a stellar generation and ejected to the interstellar medium depends on 1) the synthesis of elements by stars of various masses 2) the number of stars formed in a given interval of stellar masses i.e. on the initial mass function. We adopted the yields of the Padova group (Marigo 2000; Portinari et al. 1998) for calculation of the heavy element yields synthesized and ejected by massive, intermediate and low-mass stars during and at the end of stellar

\footnotetext{
1 This type of description of star formation rate is usually used for a numerical model. In our case "star fomation burst" means a number of stars born in a short time interval. The advantage of using this type of description is that we can obtain any type of star formation history by varying the time interval between bursts and the burst amplitude. Such a description of the star formation history is rather general and allows us to produce a star formation history in the form of bursts as a continuous star formation history.
} 
Table 1. Parameters of selected models.

\begin{tabular}{ccc}
\hline \hline Model & Thin disk & Thick disk \\
\hline$T_{\text {top }}[\mathrm{Gyr}]$ & 1 & 1 \\
$T_{\text {sfr }}[\mathrm{Gyr}]$ & 8 & 5 \\
$T_{\text {inf }}[\mathrm{Gyr}]$ & 5 & 7 \\
$a_{0}$ & 0.06 & 0.1 \\
\hline
\end{tabular}

lifetimes. The elements that have been included in the calculation are $\mathrm{He}, \mathrm{C}, \mathrm{O}, \mathrm{Mg}, \mathrm{Si}, \mathrm{S}, \mathrm{Fe}, \mathrm{Ca}$. The initial mass function is described by the Salpeter law with $A=2.35$ (Salpeter 1955). In this paper we have used the yields with above-solar initial metallicity, $Z=0.015$. Drastic differences exist between predicted yields for stars with initial metallicity $Z=0$ and $Z>0$ (Woosley \& Weaver 1995) therefore the effect of a reasonable initial metallicity on final stellar yields is negligible for stars with $Z>0$.

The Mg yield in the paper of Portinari et al. (1998) was determined from Woosley and Weaver's yields (Woosley \& Weaver 1995) which give an underestimated $\mathrm{Mg}$ value (see Thomas et al. $2000)$; therefore the $[\mathrm{Mg} / \mathrm{Fe}]$ ratio is not reproduced well in their paper (Portinari et al. 1998).

François et al. (2004) tested the Woosley and Weaver yields and found that the $\mathrm{Mg}$ yield is underestimated. In order to reproduce the $\mathrm{Mg}$ abundance there is a need to increase the predicted $\mathrm{Mg}$ yield from stars with masses $11-20 M_{\odot}$ by a factor of 7 while the yields from stars with masses of more then $20 M_{\odot}$ need be lowered about two-fold.

Making use of Portinari's data in the calculation of the model of chemical evolution we found that stars formed from a gas with the initial metallicity $Z<0.15$ give a lower $\mathrm{Mg}$ yield so as to reproduce the observed data. But stars with $Z=0.15$ give even higher $\mathrm{Mg}$ yields; we had to lower the predicted $\mathrm{Mg}$ yield from the stars with $9-15 M_{\odot}$ by a factor of 1.5 so that the model results would be in good agreement with the observation data in the $[\mathrm{Mg} / \mathrm{Fe}]$ vs. $[\mathrm{Fe} / \mathrm{H}]$ and $[\mathrm{Si} / \mathrm{Fe}]$ vs. $[\mathrm{Mg} / \mathrm{Fe}]$ diagrams.

Figure 2 demonstrates the cumulative mass of magnesium, silicon, oxygen and iron synthesized by a single stellar generation. The iron yield contributed by SNIa is taken into account in the calculation of the total $\mathrm{Fe}$ yield. It was assumed that type Ia supernovae evolve according to a SD (single degenerate) scenario, in the framework of which the accretion of $\mathrm{He}$ or $\mathrm{H}$ on a white dwarf in a binary system is considered (see Nomoto 1982; Hachisu et al. 1996). The Fe contribution from SNII and SNIa types at all times of evolution is about $1 / 3$ and $2 / 3$ correspondingly.

\section{The model results}

\subsection{The $\alpha$-element abundances}

In this paper we compared the model predictions with the observations of abundances of the thin and thick disk stars from the paper of Mishenina et al. (2004). Data of Bensby et al. (2003) (thick disk star ages) were used in addition for a more extended sample of thick disk stars. In our paper, ages for Mishenina's data set were calculated using the Bertelli isochrones (Bertelli et al. 1994).

The model predictions for the thin disk relative abundances are shown in Figs. 3, 5. The best fit model parameters are listed in Table 1. The star formation and accretion rates for the thin disk are shown in Figs. 1a,b.
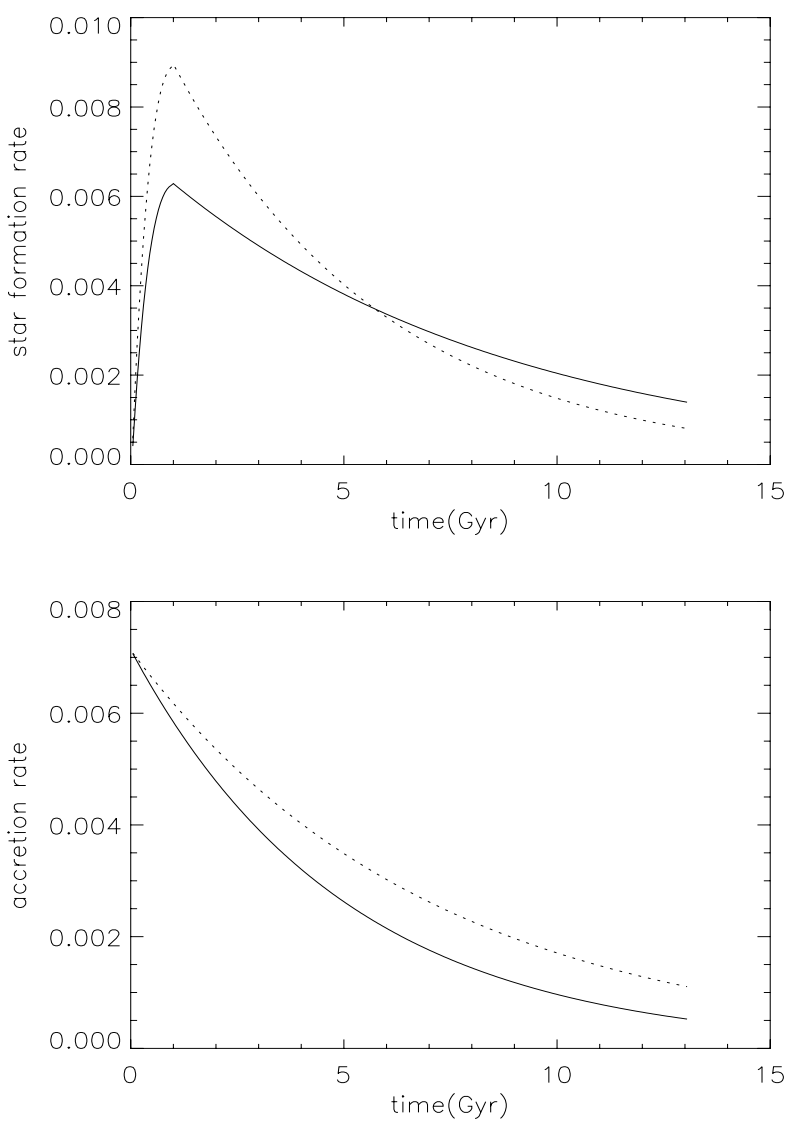

Fig. 1. The star formation and the accretion rates as a function of time in the model for thin (solid line) and thick (dashed line) disks.

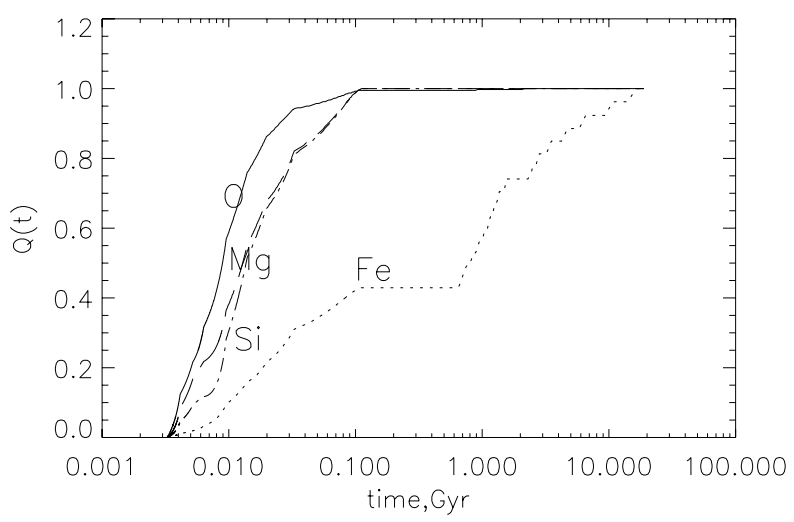

Fig. 2. The cumulative amounts of newly synthesized elements ejected by a stellar generation as a function of time. Each curve is normalized to its value at $t=13 \times 10^{9} \mathrm{yr}$.

The parameters were chosen so that the use of such parameters for the star formation and accretion rate would reproduce the observed data.

As Fig. 3 demonstrates, the use of the above-mentioned parameter values allows us to reproduce the $[\mathrm{Mg} / \mathrm{Fe}],[\mathrm{Si} / \mathrm{Fe}]$ and $[\mathrm{Mg} / \mathrm{Si}]$ thin disk ratios quite well. The model track agrees closely with the line drawn by the least-squares method through a cloud of points marking the positions of the thin disk stars in the $[\mathrm{Mg} / \mathrm{Fe}]$ vs. $[\mathrm{Fe} / \mathrm{H}]$ and $[\mathrm{Si} / \mathrm{Fe}]$ vs. $[\mathrm{Fe} / \mathrm{H}]$ diagrams. However, the model poorly reproduces the observed data at the super-solar metallicities especially for $\mathrm{Si}$.

Model predictions for the thick disk are shown in Fig. 4. The best fit parameters of thick disk evolution are presented in 

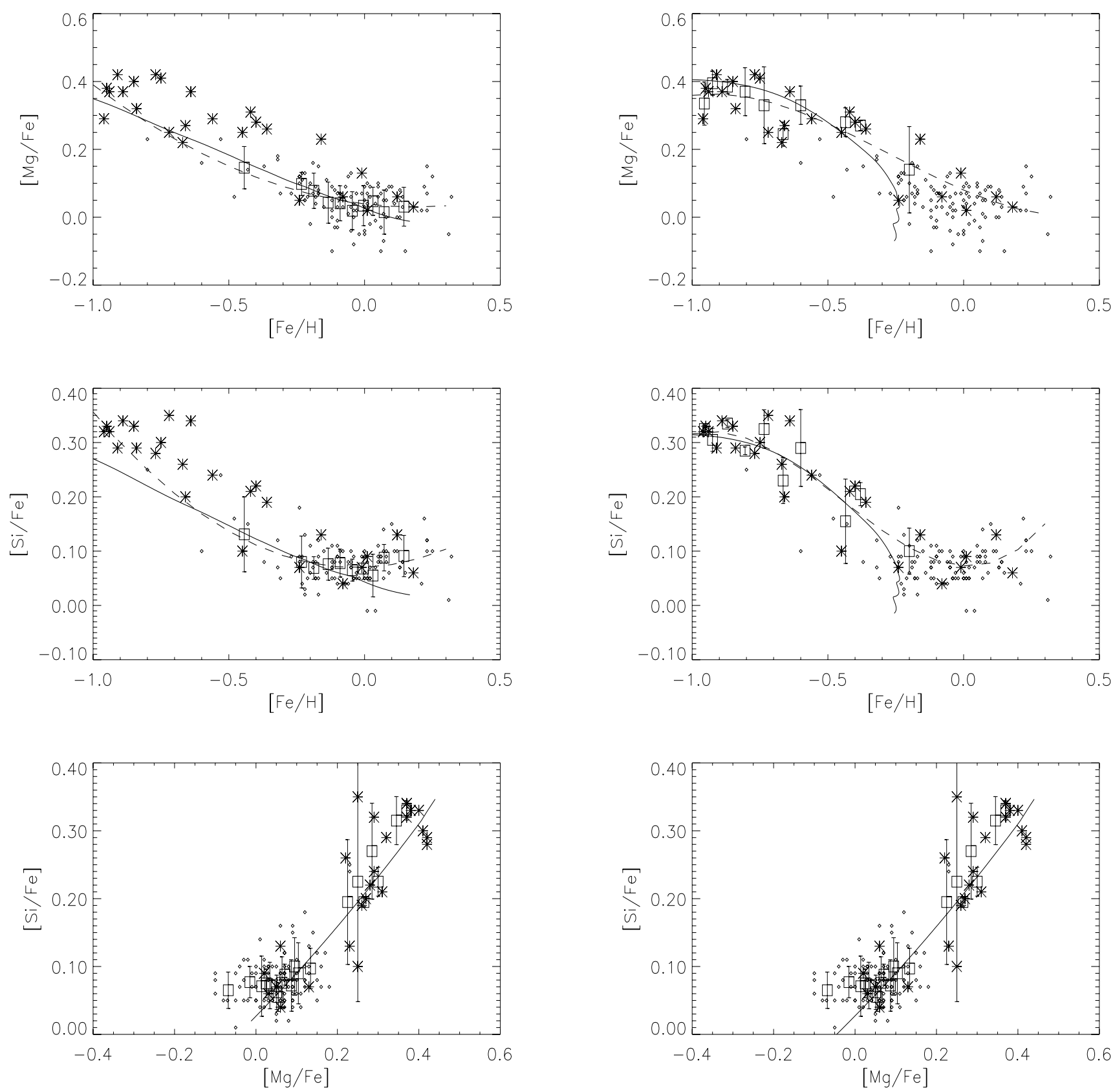

Fig. 3. The model predictions of the relative abundances of Galactic thin disk stars. The data are taken from Mishenina et al. (2004) and Bensby et al. (2003, thick disk star ages only). The asterisks are thick disk stars, the small diamonds are thin disk stars, squares are observed data averaged in 10 bins with equal numbers of stars. The dashed line is a curve drawn by the least-squares method in the observed data. The model prediction for the thin disk is indicated by a solid line.

Table 1. The star formation and accretion rates for the thick disk are shown in Figs. 1a,b. As Fig. 4 shows, the solid line of the model prediction for the thick disk is in agreement with dashed line obtained by averaging the least-squares method of the observed relative abundances of $\mathrm{Mg}, \mathrm{Si}$ and $\mathrm{Fe}$.

The model predictions for thin disk element abundances as functions of time are worse than for relative abundances. Figure 5 shows that the predicted abundance trends for the thin disk are slightly lower than Mishenina's observations averaged by the least-square method.

Fig. 4. The model predictions of the relative abundances of Galactic thick disk stars. Symbols are the same as in Fig. 3.

The obtained values of parameters of thin disk evolution are in good agreement with parameters of the best fit model of the Galactic disk of Pilyugin \& Edmunds (1996) who have investigated the "age $-[\mathrm{Fe} / \mathrm{H}]$ " and "age $-[\mathrm{O} / \mathrm{H}]$ " ratios and the solar neighbourhood metallicity distribution function. The assumed disk age is $13 \mathrm{Gyr}$; the obtained age - metallicity relation reproduces quite well the observed disk star abundances of Edvardsson et al. (1993). We compared the model prediction for the thin disk with the observed abundances of disk stars obtained by Edvardsson et al. (1993) for the whole disk. Figure 6 demonstrates that under the same parameters the thin disk modelled curve is in agreement with the observed age - metallicity relation.

Recently Nordström et al. (2004) presented new determinations of metallicities and ages of F and G-dwarfs in the solar neighbourhood. We used the probabilities of the stars with 

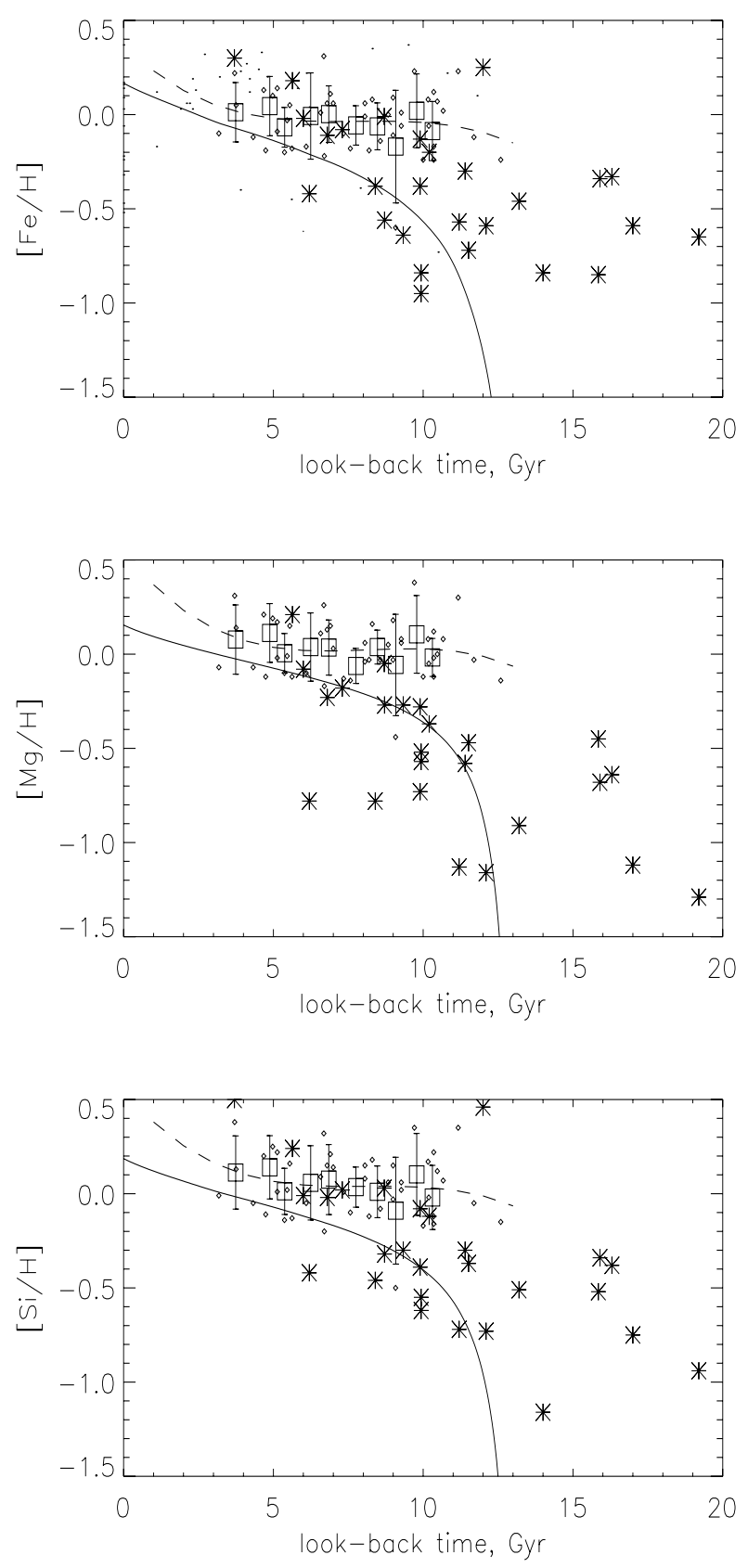

Fig. 5. The model predictions of the abundances of Galactic thin disk stars as a function of time. Symbols are the same as in Fig. 3.

measured velocities $(U, V, W)$ from the paper of Mishenina et al. (2004) and the ages and metallicities of Nordström's survey for the stars investigated in both papers to build the new "age metallicity" relation, Fig. 7 (top). Figure 7 shows that predicted abundances of the thin disk model $\left(t_{\mathrm{gal}}=13 \mathrm{Gyr}\right)$ are also lower on average, as we can see in the case of Mishenina's data.

The computed probability of belonging to thin/thick disk for stars with measured velocities $(U, V, W)$ of Mishenina et al. (2004) are in good agreement with the ones of Bensby et al. (2003). Nordström et al. (2004) have compared their photometric metallicities with the spectroscopic values for F and G-stars from Edvardsson et al. (1993) and found that the agreement is excellent with a mean difference of only 0.02 dex and dispersion around the mean of 0.08 .

Thus, there is the question of whether this "overestimated" (in comparison with the average metallicity of the whole disk)

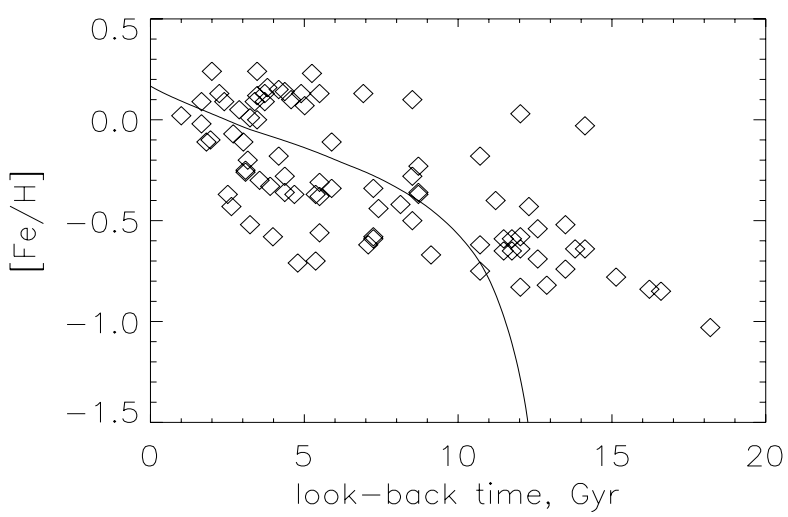

Fig. 6. The "age-metallicity" relation of the Galactic disk Data are taken from Edvardsson et al. (1993). The model prediction of the thin disk is indicated by the solid line.
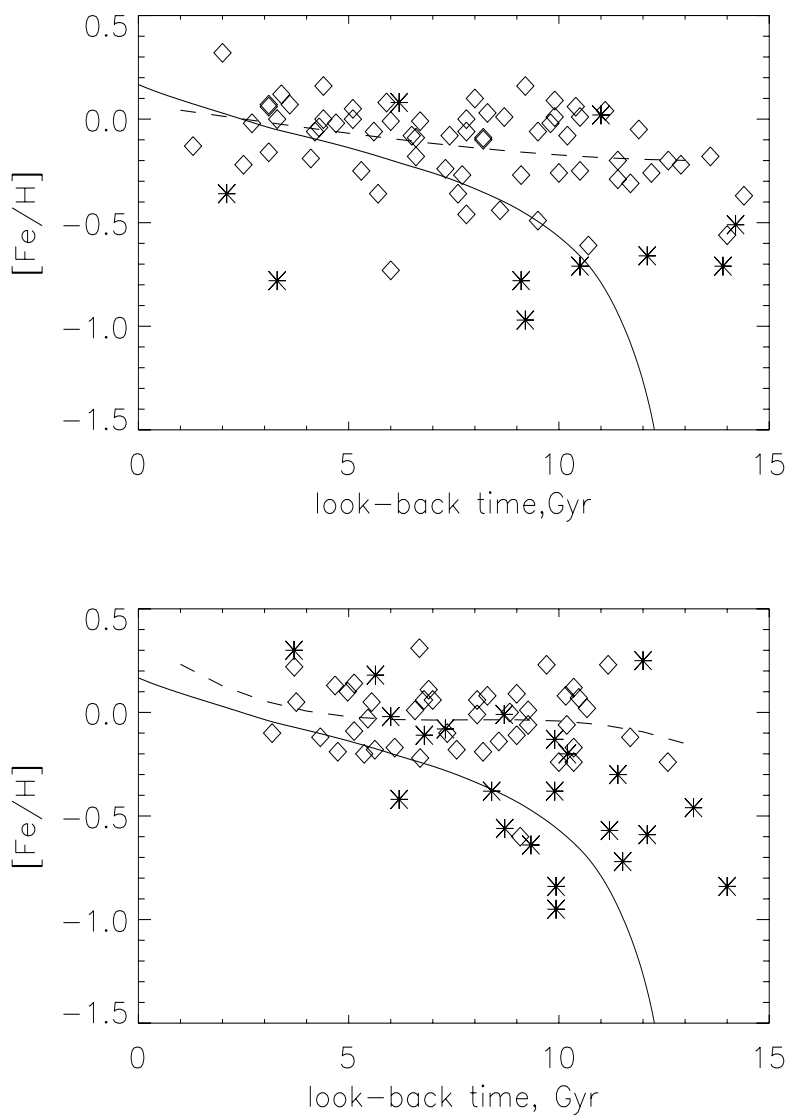

Fig. 7. The "age - metallicity" relation of the thin and thick disk of the Galaxy obtained by Nordström et al. (2004, top), and by Mishenina et al. $(2004$, bottom $)$. The thin disk stars are marked by large diamonds, thick disk stars - asterisks. The solid line is the model prediction for the thin disk, the dashed line is drawn by the least-squares method.

mean value of abundances is usual for thin disk stars. If this is the case, it needs to provide a solution to this problem since it is not enough to change only star formation and accretion parameters in order to reproduce the thin disk age - metallicity relation in the framework of our model. Such a age - metallicity relation (Fig. 5) can be explained either by thin disk pre-enrichment or an infall of metal-enriched gas. We consider that in our model the thin disk is pre-enriched as we have used stellar yields with above-solar initial metallicity. Tosi (1988) calculated the model with metal-rich gas infall for the Galactic disk. Table 2 in her 

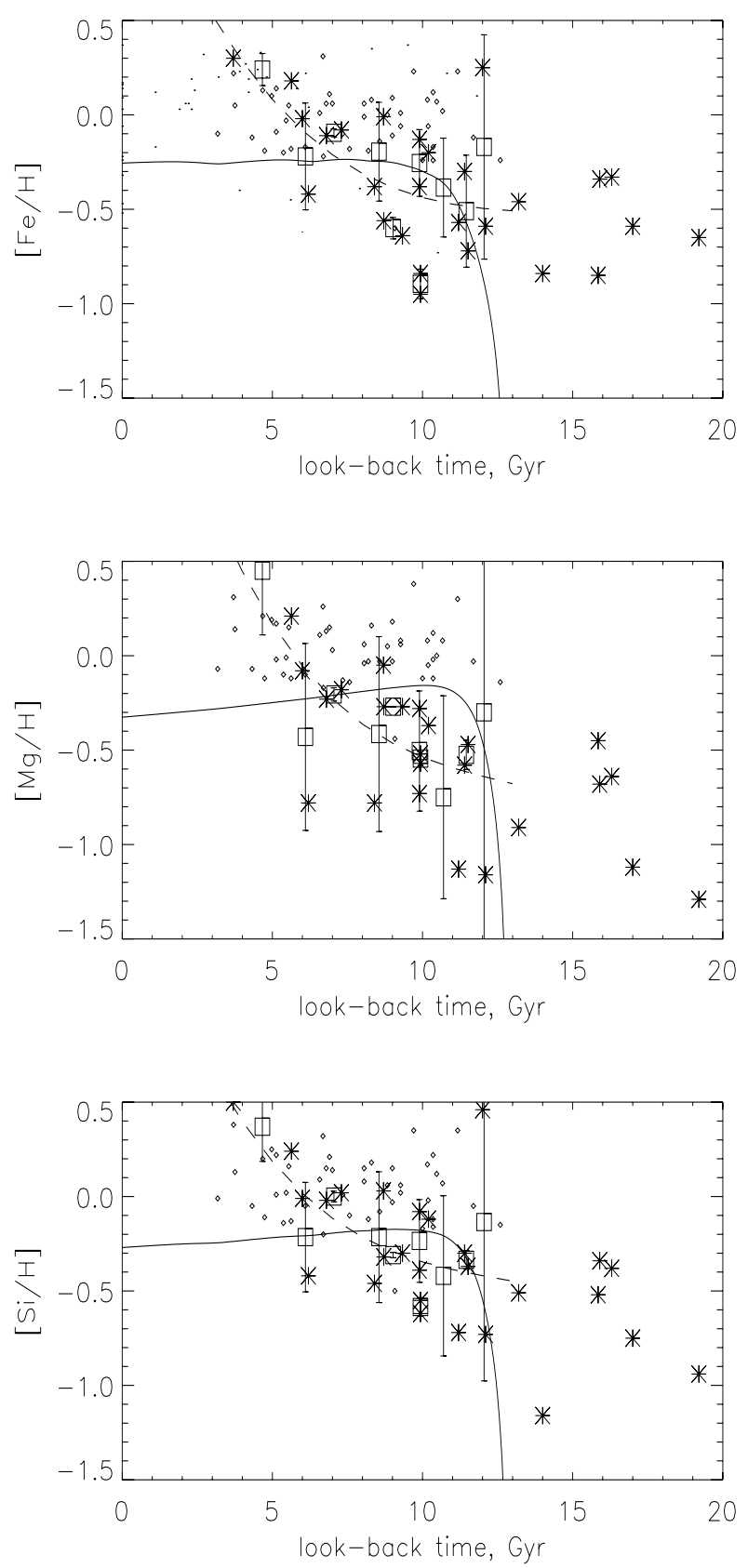

Fig. 8. The model predictions of the abundances of Galactic thick disk stars as a function of time. Symbols are the same as in Fig. 3.

paper demonstrates that the effect of a metal-rich infall on the age - metallicity relation is negligible in the solar neighbourhood. We ran a set of models with infall of enriched gas with $Z$ from $0,1 Z_{\odot}$ to $1 Z_{\odot}$. Solar proportions for the abundances of the various metals were assumed. We found out that the infall of enriched gas does not noticeably change the position of the modelled curve on the age - metallicity diagram.

Unfortunately, the number of stars belonging kinematically to the thin disk exceeds the number of stars belonging to the thick disk; among the latter there are not enough objects for which one can reliably determine the ages. Therefore, the trend in the "age - metallicity" diagram for the thick disk stars is less reliable than for the thin disk stars. The few thick disk stars with determined ages were taken from Bensby et al. $(2003,2004)$ to increase the number of thick disk stars in the "age - metallicity", "age $-[\mathrm{Mg} / \mathrm{H}]$ " and "age - $[\mathrm{Si} / \mathrm{H}]$ " diagrams.

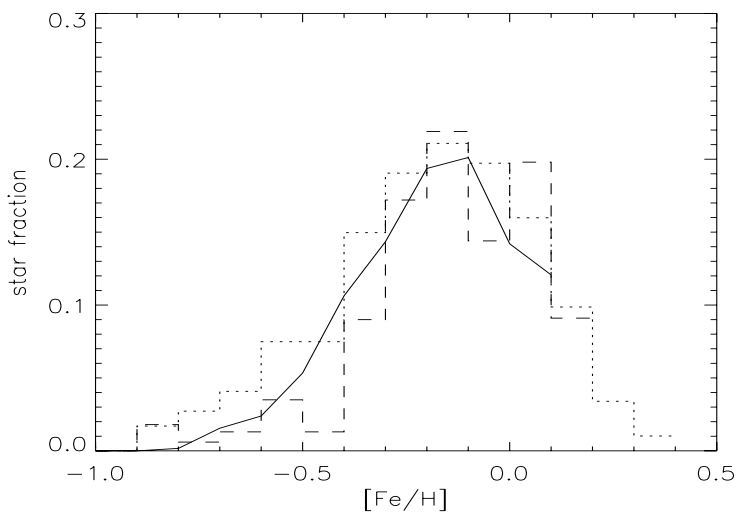

Fig. 9. The metallicity distribution function. The dotted line is the observed distribution obtained by Hou et al. (1998), the dashed line is the observed distribution obtained by Jorgensen (2000), the solid line is the model prediction for the thin disk.

The situation is slightly complicated by the presence of stars with thick disk kinematics and thin disk metallicity in the observed data of Mishenina et al. (2004). These stars are marked with asteriscs as belonging to the thick disk population but are located in the region occupied by a younger and more metal-rich thin disk population (e.g. Fig. 3).

The "age - metallicity" diagram (see Fig. 4) shows that the thick disk stars are less metal-rich and older than the thin disk stars. We have a problem determining the age range of thick disk stars. The use of various sets of isochrones gives ages of the oldest thick disk stars exceeding the age of Univers by several Gyrs.

Until the ages of the oldest stars are determined accurately, we can consider the "age - metallicity" relation of thick disk stars as only an estimate of predicted abundance ratios. Nevertheless, the predicted "age - metallicity" ratio (as "age $[\mathrm{Si} / \mathrm{H}]$ " ratio) reproduces the average values of the observed data for thick disk stars marked by squares with errors in Fig. 4.

The abundance ratio of $\mathrm{Mg}$ and $\mathrm{Si}$ depends on the yields of single stars with different masses and lifetimes used in the model. As Figs. 3 and 4 show, the $\mathrm{Mg}$ and $\mathrm{Si}$ abundance ratios are in agreement with the observed data of the thin as thick disks, except the observed data at super-solar metallicities. The behavior of the thin and thick disk abundance trends at super solar metallicities requires further study.

\subsection{The metallicity distribution function}

The successful modeling of the chemical evolution should reproduce the G-dwarf metallicity distribution function of the Galactic disk. The model prediction was compared with observations of the metallicity distribution function in the solar neighbourhood (Fig. 9). It is known that $94 \%$ of solar neighbourhood stars belong to the thin disk whereas the remaining $6 \%$ belong to the thick disk population (Robin et al. 1996). Therefore the observed metallicity distribution functions in Fig. 9 were compared with the model prediction for the thin disk. With the abovementioned parameters of the star formation and gas accretion in the thin disk (see Table 1), the model reproduces the observed metallicity distribution functions quite well (Fig. 9).

\subsection{Comparison with previous work and discussion}

A model of chemical evolution should account for the origin and characteristics of the subsystems of the Galaxy. A set of papers 
dedicated to aspects of galactic chemical evolution exists in the literature. Most of these papers deal with the standard open models of chemical evolution with inflow of intergalactic gas in the Galaxy.

Chiappini et al. (1997) considered a two-zone model for the disk and the halo, the two-infall model with two episodes of intergalactic gas infall, in the formation process of the thin disk and the halo-thick disk. Their model predicts the abundances of 16 chemical elements except $\mathrm{Mg}$ and ${ }^{3} \mathrm{He}$. The authors conclude that the comparison between theory and observations suggest a lower iron yield from SNIIs and an increased $\mathrm{Mg}$ yield from SNIIs. In the framework of Chiappini's model, François et al. (2004) have computed the chemical evolution of 12 elements (including $\mathrm{O}, \mathrm{Mg}, \mathrm{Si}$ ) and have reproduced the results of observations for $\mathrm{O}, \mathrm{Si}$ and $\mathrm{Ca}$. Their $[\mathrm{Mg} / \mathrm{Fe}]$ ratio is underestimated owing to the low $\mathrm{Mg}$ yield from massive stars. Chang et al. (1999) introduced the two-component model of chemical evolution which assumes that the thin and thick disk of the Galaxy have formed during two accretion episodes. Their models reproduce the metallicity distribution function, age metallicity relation and $[\mathrm{O} / \mathrm{Fe}]-[\mathrm{Fe} / \mathrm{H}]$ ratio for the Solar vicinity. Alibes et al. (2001) analyzed the evolution of all stable isotopes between $\mathrm{H}$ and $\mathrm{Zn}$. Their model also assumes the formation of the Galaxy in two main episodes of exponentially decreasing infall. The first episode forms the halo and the thick disk from infall of primordial extragalactic gas over $1 \mathrm{Gyr}$. The thin disk forms by infall of gas with metallicity $0.12 Z_{\odot}$ over $7 \mathrm{Gyr}$. Their model predictions of abundance ratios are in agreement with observation except for $\mathrm{Mg}$ (the authors used the yields of Woosley \& Weaver 1995).

Goswami \& Prantzos (2000) studied the evolution of the abundances of the intermediate mass elements ( $\mathrm{C}$ to $\mathrm{Zn}$ ) in the Galactic halo and local disk. Their model assumes strong outflow in the halo phase and infall in the disk, which allows them to reproduce the corresponding metallicity distribution. The evolution of $\alpha$-elements $\mathrm{O}, \mathrm{Si}, \mathrm{S}$ and $\mathrm{Ca}$ is well understood with the assumption that SNIa contribute most of the Fe in the disk but the Mg is underproduced in the Woosley \& Weaver (1995) yields, therefore their $\mathrm{Mg}$ yield is relative to the observation in the disk. The model predictions cover a wide $[\mathrm{Fe} / \mathrm{H}]$ range, from -4.0 up to 0.0 , but do not show the clear limits of the model components. One can define the limits only by comparing their predictions with observations. Unfortunately, in the abovementioned papers model predictions have not be compared with observations for the thin and thick disks separately and we can only conclude about the evolution of the disk as whole.

In contrast, we consider the thick disk evolution separately from the halo evolution. The new observations demonstrate the clear separation of Galaxy disk stars into the thin and thick disk population in the range $-0.6<[\mathrm{Fe} / \mathrm{H}]<-0.3$ (see Introduction) that shows the need to consider the Galactic thick disk evolution separately, not only from the thin disk evolution but also from the halo evolution.

Ferrini et al. (1992) have considered the evolution of the solar neigbourhood as subdivided into halo and disk regions. The chemical abundances of 14 species (in particular $\mathrm{O}, \mathrm{Mg}, \mathrm{Si}$, $\mathrm{Fe}$ ) have been predicted for the disk and halo. Their $[\mathrm{O} / \mathrm{Fe}]$ vs. $[\mathrm{Fe} / \mathrm{H}]$ diagram demonstrates the predicted model curve covering the $[\mathrm{Fe} / \mathrm{H}]$ range of -2.5 to $0.5(0.0<[\mathrm{O} / \mathrm{Fe}]<0.5)$ for the disk and -2.5 to -1 . $(0.2<[\mathrm{O} / \mathrm{Fe}]<0.5)$ for the halo. Travaglio et al. (1999) have calculated the Galactic chemical evolution of the elements from $\mathrm{Ba}$ to $\mathrm{Eu}$ in the framework of the three-zone model of chemical evolution (the halo, the thin disk, the thick disk). Later, the Galactic evolution of $\mathrm{Pb}$,

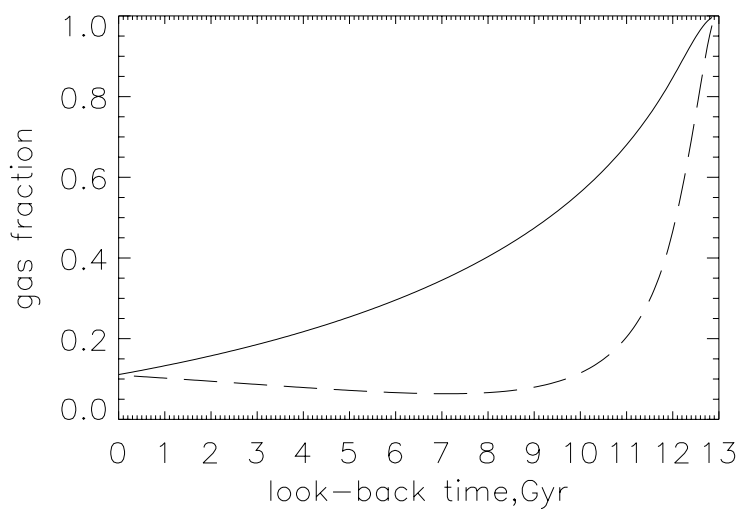

Fig. 10. The gas fraction as a function of time in the thin (solid line) and thick (dashed line) disk computed by the models.

$\mathrm{Cu}$ and $\mathrm{Zn}$ was computed in framework of the same model (Travaglio et al. 2001; Mishenina et al. 2002). As an example of the model results, Travaglio et al. (1999) computed the values of $[\mathrm{O} / \mathrm{Fe}]$ vs. $[\mathrm{Fe} / \mathrm{H}]$ in the three Galactic zones of their standard model. We can see from their Fig. $3 b$ that the thick disk phase covers the interval $-2.5 \lesssim[\mathrm{Fe} / \mathrm{H}] \lessgtr-1$, and the thin disk phase begins at $[\mathrm{Fe} / \mathrm{H}] \gtrsim-1.5$. However, the new observations show clearly that the thick disk star population continues up to $[\mathrm{Fe} / \mathrm{H}]=-0.3$ at least (Mishenina et al. 2004). According to Mishenina et al. (2004), star formation in the thick disk stopped when the enrichment was $[\mathrm{Fe} / \mathrm{H}]=-0.3,[\mathrm{Mg} / \mathrm{Fe}]=+0.2$ and $[\mathrm{Si} / \mathrm{Fe}]=+0.17$. Our model predicts that the thick disk relative abundances (Fig. 4) come abruptly to an end at $[\mathrm{Fe} / \mathrm{H}] \sim-0.3$ and deviate significantly from the least-square method curve at $[\mathrm{Mg} / \mathrm{Fe}] \sim+0.2$ and $[\mathrm{Si} / \mathrm{Fe}]=+0.17$. In our case the thick disk star formation stopped at similar values as noticed by Mishenina et al. (2004). But the observations show that there are a few thick disk stars with $[\mathrm{Fe} / \mathrm{H}]>-0.3$ (see also Bensby et al. 2005). Our model does not predict the formation of these stars in the thick disk. It is possible that these stars were not formed in the thick disk but were born in a galaxy - satellite and accreted into the Galactic disk.

Looking at the observed ratios $[\mathrm{O} / \mathrm{Fe}]$ vs. $[\mathrm{Fe} / \mathrm{H}]$ and $[\mathrm{Fe} / \mathrm{O}]$ vs. $[\mathrm{O} / \mathrm{H}]$ in the thick and thin disks, Bensby et al. (2004) concluded that the thin and thick disks were formed in different epochs and from a homogeneous gas. In their view, the star formation rate in the thick disk needs to be rapid to contribute the oxygen by SNII at the high metallicities before enrichment by SNIa (which do not contribute oxygen) will begin. Yet, the thin disk evolution must be slow and the star formation rate low. This conclusion is in good agreement with results of the two-infall model of Chiappini et al. (1997). Their model predicts a timescale of 1 Gyr for the thick disk and halo formation and about $8 \mathrm{Gyr}$ for the thin disk formation. Their model successfully reproduced $[\alpha / \mathrm{Fe}]$ vs. $[\mathrm{Fe} / \mathrm{H}]$ for the Galactic thick diskhalo and the thin disk. But they did not compare their results with observed thin and thick abundance disk trends separately since at that time such observations had not been obtained yet.

An indicator of star formation history in the model is the "gas fraction - time" relation since it indicates what fraction of galactic gas was converted into stars and during what time it occured. We found that the star formation history for the thick and thin disks must be different to reproduce the observed abundance trends. The obtained "gas fraction - time" relation indicates that the star formation history of the thick disk was rather brief as the majority of the thick disk stars was formed 2-3 Gyr and had 
almost stopped more then 10 Gyr ago (Fig. 10). On the contrary, the thin disk stars only began to form 9-10 Gyr ago (Fig. 10) and the star formation in the thin disk has decreased and is now almost stopped.

The accretion of intergalactic gas significantly affects the abundance predictions for the disk subsystems of our Galaxy. The observations show that the thin disk and thick disk exhibit parallel slopes of $[\alpha / \mathrm{Fe}]$ vs. $\mathrm{Fe} / \mathrm{H}]$ in the range $-0.8<[\mathrm{Fe} / \mathrm{H}]<$ -0.3 . It is supposed that the slope reflects the contribution of different supernovae to the interstellar medium enrichment. In our paper, the Fe yield from SNIa is taken into account both for the thin and thick disks since the thick disk stars show evidence of chemical enrichment from SNIa (Mishenina et al. 2004; Bensby et al. 2003). The difference between the thick and thin disk abundance trends is caused by an increased inflow of gas mass in our model. The trend of the relative abundances of the thin disk stars will change location and approach the region occupied by the thick disk stars in the range of $-1.0<[\mathrm{Fe} / \mathrm{H}]<$ -0.3 if the mass of infalling gas at each accretion episode increases significantly (more than a factor of 10). In other words, the $\alpha$-element enhancement of the thick disk as compared to the thin disk can be caused by more mass inflow in a unit of time in the thick disk.

\section{Conclusions}

The chemical evolution of the disk of the Galaxy was investigated in the framework of the two-zone open model with gas inflow. It was supposed that the Galactic disk is divided into two zones - thin and thick disks, differing from each other chemically and having different evolution timescales.

The Galactic evolution of $\alpha$-elements $(\mathrm{Mg}$ and $\mathrm{Si}$ ) and $\mathrm{Fe}$ was predicted for the thin and thick disks and was compared with recent observations. Our model predicts that the thick disk star formation stopped at $[\mathrm{Fe} / \mathrm{H}] \sim-0.3$ and at $[\mathrm{Mg} / \mathrm{Fe}] \sim+0.2$ and $[\mathrm{Si} / \mathrm{Fe}]=+0.17$; for the thin disk relative abundances our model predictions cover the interval $-1.0<[\mathrm{Fe} / \mathrm{H}]<0.0$, that agrees with observations.

We have obtained the values of parameters of the star formation history for the thin and thick Galactic disks and conclude that it is necessary to use different star formation histories for the thick and thin disks to reproduce the observed abundance trends.

Gas infall plays an important role in the appearance of $\alpha$-element enhancement of the thick disk compared to the thin disk - the inflow rate must be more intensive per unit of time for the thick disk than the thin disk of the Galaxy.

Acknowledgements. We thank Dr. L. S. Pilyugin, Dr. C. Travaglio and especially the referee, Dr. François, for helpful discussions and useful comments.

\section{References}

Alibes, A., Labay, J., \& Canal, R. 2001, A\&A, 370, 1103 Bensby, T., Feltzing, S., \& Lundstrom, I. 2003, A\&A, 410, 527
Bensby, T., Feltzing, S., \& Lundstrom, I. 2004, Origin and evolution of elements, from the Carnegie Observatories Centenial Symposia. Carnegie Observatories Astrophysics Series, ed. Mc William, \& M. Rauch

Bensby, T., Feltzing, S., \& Lundstrom, I. 2004, A\&A, 415, 155

Bensby, T., Feltzing, S., Lundstrom, I., \& Ilyin, I. 2005, A\&A, 433, 185

Bernkopf, J., Fielder, A., \& Fuhrmann, K. 2001, in Astrophysical Ages and Timescales, ed. T. von Hippel, N. Manset, \& C. Simpson (San Francisco: ASP), 245, 207

Bertelli, G., Bressan, A., Chiosi, C., Fagotto, F., \& Nasi, E. 1994, A\&AS, 106, 275

Chang, R. X., Hou, J. L., \& Shu, C. C. 1999, A\&A, 350, 38

Chen, B. 1997, ApJ, 491, 181

Chen, Y. Q., Nissen, P. E., Zhao, G., Zhang, H. W., \& Benoni, T. 2000, A\&AS, 141,491

Chiappini, C., Matteucchi, F., \& Gratton, R. 1997, ApJ, 477, 765

Cowan, J. J., Thielemann, F.-K., \& Truran, J. W. 1991, ARA\&A, 29, 447

Edvardsson, B., Andersen, J., Gustafsson, B., et al. 1993, A\&A, 275, 101

Feltzing, S., Bensby, T., \& Lundstrom, I. 2003, A\&A, 397, L1

Ferrini, F., Matteucci, F., Pardi, C., \& Penco, U. 1992, ApJ, 387, 138

François, P., Matteucci, F., Cayrel, R., et al. 2004, A\&A, 421, 613

Fuhrmann, K. 1998, A\&A, 338, 161

Gilmore, G., \& Raid, N. 1983, MNRAS, 202, 1025

Gilmore, G., Wyse, R. F. G., \& Jones, J. B. 1995, AJ, 109, 1095

Goswami, A., \& Prantzos, N. 2000, A\&A, 359, 191

Gratton, R., Caretta, E., Matteucci, F., \& Sneden, C. 1996, in Formation of the Galactic Halo ...inside and out, ed. H. Morrison, \& A. Sarajedini (San Francisco: ASP), 92, 307

Gratton, R., Caretta, E., Matteucci, F., \& Sneden, C. 2000, A\&A, 358, 671

Hachisu, I., Kato, M., \& Nomoto, K. 1996, ApJ, 470, L97

Hou, J., Chang, R., \& Fu, C. 1998, 1997 Pacific Rim Conference on Stellar Astrophysics ASP Conf. Ser., 138, 143

Jimenez, R., Flynn, C., \& Kotoneva, E. 1998, MNRAS, 299, 515

Jorgensen, B. R. 2000, A\&A, 363, 947

Layden, A. C. 1995, AJ, 110, 2288

Layden, A. C. 1995, AJ, 110, 2312

Mashonkina, L., \& Gehren, T. 2000, A\&A, 364, 249

Mashonkina, L., \& Gehren, T. 2001, A\&A, 376, 232

Marigo, P. 2001, A\&A, 370, 194

Matteucci, F. 1992 Mem. Soc. Astron. It., 63, 301

Matteucci, F., \& Greggio, L. 1986, A\&A, 154, 279

Mishenina, T. V., Kovtyukh, V. V., Soubiran, C., Travaglio, C., \& Busso, M. 2002, A\&A, 396, 189

Mishenina, T. V., Soubiran, C., Kovtyukh, V. V., \& Korotin, S. A. 2004, A\&A, 418,551

Nissen, P. E., Chen, Y. Q., Schuster, W. J., \& Zhao, G. 2000, A\&A, 353, 722

Nomoto, K. 1982, ApJ, 253, 798

Nordström, B., Mayor, M., Andersen, J., et al. 2004, A\&A, 418, 989

Pilyugin, L. S. 1993, A\&A, 277, 42

Pilyugin, L. S. 1994, AZh, 71, 825

Pilyugin, L. S., \& Edmunds, M. G. 1996, A\&A, 313, 783

Portinari, L., Chiosi, C., \& Bressan, A. 1998, A\&A, 334, 505

Prochaska, J. X., \& McWilliam, A. 2000, ApJ, 537, L57

Prochaska, J. X., Naumov, S., Carney, B. W., McWilliam, A., \& Wolfe, A. M. 2000, AJ, 120, 2513

Reyle, C., \& Robin, A. C. 2001, A\&A, 373, 886

Robin, A. C., Haywood, M., Creze, M., Ojha, D. K., \& Bienaume, O. 1996, A\&A, 305, 125

Salpeter, E. E. 1955, ApJ, 121, 161

Soubiran, C., Bienaume, O., \& Siebert, A. 2003, A\&A, 398, 141

Thomas, D., Greggio, L., \& Bender, R. 2000, in The evolution of the Milky way: stars vs. clusters, ed. F. Matteucci, \& F. Giovanelli, 541

Tosi, M. 1988, A\&A, 197, 47

Tosi, M. 2003 [arXiv: astro-ph/0308463]

Travaglio, C., Galli, D., Gallino, R., et al. 1999, ApJ, 521, 691

Travaglio, C., Gallino, R., Busso, M., \& Gratton, R. 2001, ApJ, 549, 346

Woosley, S. E., \& Weaver, T. A. 1995, ApJS, 101, 181

Wyse, R. F. G., \& Gilmore, G. 1995, AJ, 110, 2771 